\title{
A deformation-dependent soil-water characteristic surface model considering hysteresis
}

\author{
Cai Guoqing ${ }^{1,2,{ }^{*}}$, Wu Tianchi $^{2}$, Li $\mathrm{Hao}^{3}$, Zhao Chenggang ${ }^{1,2}$, Tian Jingjing ${ }^{2}$, Li Jian ${ }^{1,2}$ \\ 1. Key Laboratory of Urban Underground Engineering, Ministry of Education, Beijing Jiaotong University, Beijing 100044, China \\ 2. School of Civil Engineering, Beijing Jiaotong University, Beijing 100044, China \\ 3. Henan Provincial Department of Transportation, Zhengzhou Henan 450000, China
}

\begin{abstract}
The soil-water characteristic surface model plays an essential part in predicting the hydraulic behaviour of unsaturated soils. Based on the theory of plasticity bounding surface, this paper presents a three-dimensional soil-water characteristic surface model considering the effects of deformation and hysteresis. Suction and void ratio are adopted as independent variables, while the degree of saturation is adopted as a dependent variable. A new mapping rule is used where the distance between the current position and its image point can be calculated as a difference in the degree of saturation axis. The model is verified by comparing with drying-wetting tests on bentonite/kaolin mix and pearl clay. The efficiency of the proposed model is proven by validation tests.
\end{abstract}

\section{Introduction}

Soil-water characteristic curve (SWCC) is adopted to represent the relationship between water content and suction of unsaturated soils. SWCC is also a primary method for studying problems about seepage, deformation, strength or other mechanical and hydraulic behaviours of unsaturated soil[1-4]. Early work on SWCC normally assumes that water content and suction share a unique correspondence[5-7]. Hysteresis, however, leads to the phenomenon that degree of saturation/volumetric strain and suction are not one-to-one correspondence during cyclical wetting and drying processes[8-12]. Besides, Collapsible loess and expansive clay usually generate nonnegligible deformation in practical cases, which brings about the change of degree of saturation. Deformation and hysteresis make mechanical and hydraulic behaviours of unsaturated soils more complex than previously expected[13-15]. Confronted with these problems, hardly can a SWCC model based on the assumption of one-to-one correspondence between suction and water content precisely describe the water capacity of unsaturated soils. In fact, a three-dimensional SWCC model considering the effects of deformation and hysteresis is more reasonable and preferable.

Recent investigations into SWCC are abundant. Zhai obtained SWCC variables by determining the mathematic relationship between SWCC parameters and fitting parameters[16]. After discovering that air entry value varies depending on specific volume, Gallipoli proposed a relationship between air entry value and specific volume then established a deformation-dependent soil-water characteristic surface model based on VG model in the $S_{\mathrm{r}}: e: v$ space[17]. Nuth found that there exists an intrinsic shape of
SWCC at a constant void ratio[18]. Only the position of SWCC will shift at different void ratios while its shape remains unchanged. Therefore, Nuth proposed a SWCC model considering deformation based on the connection between air entry value and volume change. Cyclical wetting-drying tests and loading-unloading tests on two kinds of remoulded soils and two categories of compacted soils conducted by Tarantino uncover the dependence of the main drying/wetting curve upon void ratio, so Tarantino established a relationship between water ratio and suction according to VG model and proposed void ratio-dependent equations for the main drying/wetting surface[19]. Salager performed drying tests on five samples of clayey silt with distinctive initial void ratios and established a threedimensional main drying surface in the w:s:e space on the basis of Fredlund \& Xing model[20]. According to these academic achievements, it is obvious that enormous development of SWCC models considering deformation has been achieved. However, these models all fail to take effects of hysteresis into consideration, which means they are still incapable of representing comprehensive characteristics of unsaturated soils.

$\mathrm{Hu}$ adopted the pore size distribution at a deformed state along with that at a reference state and then deduced a soilwater characteristic surface which considers deformation and hysteresis in the $e: \ln s: S_{\mathrm{e}}$ space[21]. Tsiampousi introduced the concept of bounding suction, considered the effect of specific volume, and assumed the scanning line is an arc which shares the same tangent with the main drying/wetting curve[22]. Then Tsiampousi deduced the equations of the main drying/wetting curve and the scanning curve respectively in the bounding suction:degree of saturation space. Tsiampousi also established a threedimensional soil-water characteristic surface considering

\footnotetext{
$\overline{{ }^{*} \text { Corresponding author: guoqing.cai@ } @ \text { bjtu.edu.cn }}$
} 
hysteresis in the $S_{\mathrm{r}}: s: v$ space. Although these papers mentioned above all consider the impact of deformation and hysteresis, none of them refers to the path and shape of the scanning curve in the three-dimensional space, and the validation of these models is merely conducted in a twodimensional projection plane instead of intuitively presenting true paths of the main drying/wetting curve and the scanning curve under the control of void ratio.

Overall, different models for describing hydraulic behaviours of unsaturated soils have been obtained based on multiple soils, diverse respects and various parameters. However, these models are not perfectly established and further investigation in SWCC is needed. Dafalias believed that plastic strain increment depends on the distance between the stress point in the loading surface and its corresponding image point in the bounding surface, which is called the bounding surface plasticity theory[23].

Based on the bounding surface plasticity theory, this paper establishes a three-dimensional soil-water characteristic surface model considering deformation and hysteresis. This paper also presents the shape of the main drying/wetting curve and the scanning curve in both twodimensional planes and three-dimensional space. The model adopts suction and void ratio as independent variables and degree of saturation as a dependent variable. The model prediction is compared with published experimental results of pearl clay for validation.

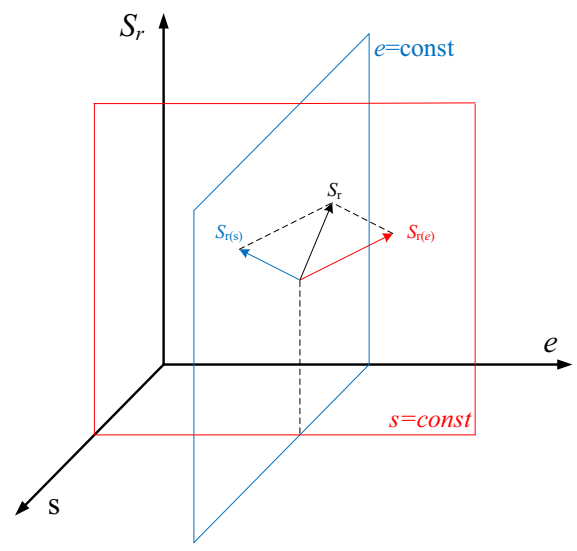

Fig.1. The decomposition of degree of saturation $S_{\mathrm{r}}$.

\section{Formulation of model deformation and hysteresis}

\section{considering}

This paper establishes a soil-water characteristic surface model considering deformation and hysteresis. Bounding surface $F$ is constituted by the main wetting surface and the main drying surface in the $S_{\mathrm{r}}: s: e$ space and soil-water characteristic curve is actually a curve formulated by a specific path in the bounding surface. This paper focuses on the variation of soil-water characteristic curve instead of the deformation of solid phase, which means only impacts of the deformation of solid phase on the SWCC is considered. It is also assumed that the increment of degree of saturation $S_{\mathrm{r}}$ can be separated into two parts: (1) the increment of $S_{\mathrm{r}}$ triggered by the change of suction $s$, which considers hysteresis and can be observed in the $S_{\mathrm{r}}$ : $s$ plane; (2) the increment of $S_{\mathrm{r}}$ brought by the change of void ratio $e$, which considers deformation and can be observed in the $e: S_{\text {r }}$ plane. The specific decomposition of $S_{\mathrm{r}}$ is shown in Fig.1.

\subsection{Bounding surface}

Tarantino performed cyclical wetting-drying tests and loading-unloading tests on two kinds of remoulded soils and two categories of compacted soils[19]. The equation of the void ratio-dependent main drying/wetting surface is proposed as follows:

$$
\left(S_{\mathrm{r}}\right)_{\mathrm{d} / \mathrm{w}}=\left\{1+\left[\left(\frac{e}{a_{\mathrm{d} / \mathrm{w}}}\right)^{1 / b_{\mathrm{d} / \mathrm{w}}} s\right]^{n_{\mathrm{d} / \mathrm{w}}}\right\}^{-b_{d / \mathrm{w}} / n_{\mathrm{d} / \mathrm{w}}}
$$

where subscripts $\mathrm{d}$ and $\mathrm{w}$ represent the drying process and the wetting process, respectively; $a, b, n$ are fitting parameters, and $a$ is related to air entry value. Values of $a_{\mathrm{d} / \mathrm{w}}$ and $b_{\mathrm{d} / \mathrm{w}}$ are equal to the intercept and slope of the 'Ins-ln $e_{\mathrm{w}}$ ' line, where $e_{\mathrm{w}}=e \cdot S_{\mathrm{r}}$ and parameter $n_{\mathrm{d} / \mathrm{w}}$ is the optimal value obtained by the method of least squares.

\subsection{Mapping rule}

According to the bounding surface plasticity theory, plastic response in the loading surface depends on the distance between the current position in the loading surface and its corresponding image point in the bounding surface. Li[24] proposed a model based on bounding surface plasticity theory for describing hysteresis during cyclical dryingwetting processes. The incremental relationship between suction on the bounding surface and suction in the scanning curve is defined as:

$$
d s=\left(\frac{\bar{s}-\alpha}{s-\alpha}\right)^{\beta} \mathrm{d} \bar{s}
$$

where $s$ is the suction of the current state; $\bar{s}$ is the suction of image point in the bounding drying/wetting curve; $\alpha$ refers to the suction corresponding to the projection centre. $\mathrm{Li}$ reckoned that $\alpha$ remain unchanged until the wetting process converts into the drying process, or vice versa, which means the projection centre is fixed for every single scanning curve. However, Wei indicated that undesirable rigidity occurs if the fixed projection centre is adopted to predict the scanning curve[25]. Therefore, similar to Wei's model, this paper still assumes that the projection centre moves along with the current position in the bounding curve. Then the former equation (2) can be written as:

$$
\hat{K}_{\mathrm{p}}=\left(\frac{r}{r-\varphi}\right)^{\beta} \bar{K}_{\mathrm{p}}
$$

where $\hat{K}_{\mathrm{p}}$ and $\bar{K}_{\mathrm{p}}$ are plastic moduli for the scanning curve and for the bounding curve, respectively, $\beta$ is a material parameter related to the size of the hysteresis loop, $r$ is the size for the bounding zone, and $\varphi=|s-\bar{s}|$ refers to the distance between the current position and its image point.

It should be noticed that the distance between the current position and its image point is normally calculated as a 
difference in the suction axis [24-26] because the authors all adopted water content as an independent variable to analyse the change of suction. According to the conversion rule among soil's three phases that $S_{\mathrm{r}}=(w / e) G_{\mathrm{s}}$, both the change of void ratio and water content can lead to the variation of degree of saturation, which intrinsically represents the change of water phase. Therefore, this paper finds out a new method to establish a hysteresis-related model that we calculate the difference in the degree of saturation axis, adopting degree of saturation as a dependent variable and suction and void ratio as independent variables. Then the final mapping rule is adopted as follows:

$$
\hat{K}_{\mathrm{p}}=\left(\frac{r-\varphi}{r}\right)^{\beta} \bar{K}_{\mathrm{p}}
$$

where $\varphi=|s-\bar{s}|$ refers to the distance between the current position and its image point.

\subsection{Scanning curve}

In the $s: S_{\mathrm{r}}$ projection plane, first consider the drying process. As shown in Fig.2, AB is the drying scanning curve, where $A$ is the current position. $O$ is the projection centre and $A^{\prime}$ is the image point in the main drying bounding curve. Point $A$, $\mathrm{A}^{\prime}$ and $\mathrm{O}$ share the same suction. The degree of saturation of $\mathrm{A}^{\prime}$ and that of $\mathrm{O}$ correspond to $S_{\mathrm{rd}}$ in the main drying bounding curve and $S_{\mathrm{rw}}$ in the main wetting bounding curve, respectively.

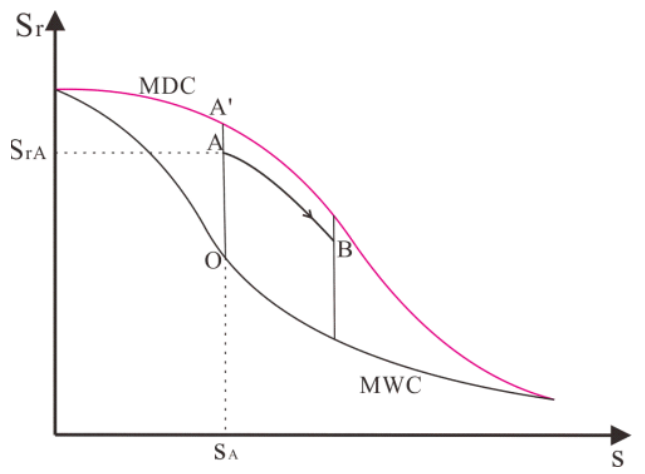

Fig.2. a drying scanning curve in $\left(s, S_{\mathrm{r}}\right)$ projection plane.

Substituting void ratios of each current position into the equation for bounding surface, the algebraic main drying/wetting surface can be obtained:

$$
\begin{aligned}
& \left(S_{\mathrm{r}}\right)_{\mathrm{d}}=\left\{1+\left[\left(\frac{e}{a_{\mathrm{d}}}\right)^{1 / b_{\mathrm{d}}} s\right]^{n_{\mathrm{d}}}\right\}^{-b_{\mathrm{d}} / n_{\mathrm{d}}} \\
& \left(S_{\mathrm{r}}\right)_{\mathrm{w}}=\left\{1+\left[\left(\frac{e}{a_{\mathrm{w}}}\right)^{1 / b_{\mathrm{w}}} s\right]^{n_{\mathrm{w}}}\right\}^{-b_{\mathrm{w}} / n_{\mathrm{w}}}
\end{aligned}
$$
is:

The slope of the main drying bounding curve at point $\mathrm{A}^{\prime}$

$$
\begin{aligned}
\bar{K}_{\mathrm{pd}}^{s} & =\frac{\mathrm{d} S_{r}}{\mathrm{~d} s} \\
& =-b_{\mathrm{d}} \cdot\left(\frac{e}{a_{\mathrm{d}}}\right)^{1 / b_{\mathrm{d}}} \cdot\left[\left(\frac{e}{a_{\mathrm{d}}}\right)^{1 / b_{\mathrm{d}}} \cdot s\right]^{n_{\mathrm{d}}-1} \cdot\left\{1+\left[\left(\frac{e}{a_{\mathrm{d}}}\right)^{1 / b_{\mathrm{d}}} \cdot s\right]^{n_{\mathrm{d}}}\right\}^{-b_{\mathrm{d}} / n_{\mathrm{d}}-1}
\end{aligned}
$$

According to the mapping rule, the slope of the scanning curve at point $\mathrm{A}$ can be obtained:

$$
\hat{K}_{\mathrm{pd}}^{s}=\left(\frac{r-\varphi}{r}\right)^{\beta} \bar{K}_{\mathrm{pd}}^{s}=\left(\frac{S_{r \mathrm{~A}}-S_{r \mathrm{O}}}{S_{r_{\mathrm{A}}}-S_{r \mathrm{O}}}\right)^{\beta} \bar{K}_{\mathrm{pd}}^{s}=\left(\frac{S_{r \mathrm{~A}}-S_{r \mathrm{w}}}{S_{r \mathrm{~d}}-S_{r \mathrm{~W}}}\right)^{\beta} \bar{K}_{\mathrm{pd}}^{s}
$$

Consider the wetting process. As shown in Fig.3, CD is the wetting scanning curve, where $\mathrm{C}$ is the current position. $\mathrm{O}$ is the projection centre and $\mathrm{C}^{\prime}$ is the image point in the main drying bounding curve. Point $\mathrm{C}, \mathrm{C}^{\prime}$ and $\mathrm{O}$ share the same suction. The degree of saturation of $\mathrm{C}^{\prime}$ and that of $\mathrm{O}$ correspond to $S_{\text {rd }}$ in the main wetting bounding curve and $S_{\text {rw }}$ in the main wetting bounding curve.

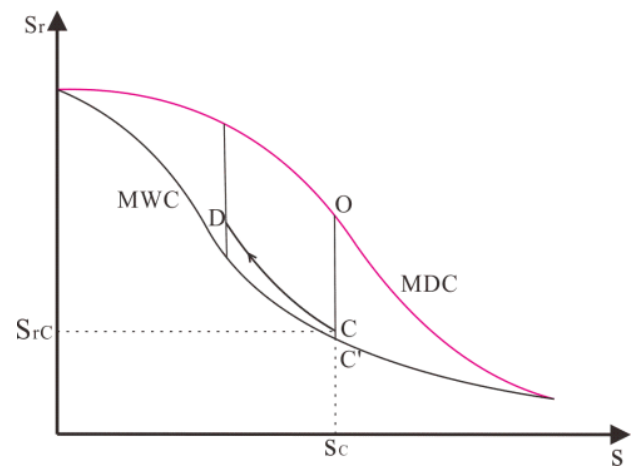

Fig.3. a wetting scanning curve in $\left(\mathrm{s}, S_{\mathrm{r}}\right)$ projection plane.

The slope of the main wetting bounding curve at point $\mathrm{C}^{\prime}$ is:

$$
\begin{aligned}
& \bar{K}_{\mathrm{pw}}^{s}=\frac{\mathrm{d} S_{r}}{\mathrm{~d} s} \\
& =-b_{\mathrm{w}} \cdot\left(\frac{e}{a_{\mathrm{w}}}\right)^{1 / b_{\mathrm{w}}} \cdot\left[\left(\frac{e}{a_{\mathrm{w}}}\right)^{1 / b_{\mathrm{w}}} \cdot s\right]^{n_{\mathrm{w}}-1} \\
& \cdot\left\{1+\left[\left(\frac{e}{a_{\mathrm{w}}}\right)^{1 / b_{\mathrm{w}}} \cdot s\right]^{n_{\mathrm{w}}}\right\}^{-b_{\mathrm{w}} / n_{\mathrm{w}}-1}
\end{aligned}
$$

According to the mapping rule, the slope of the scanning curve at point $\mathrm{C}$ can be obtained:

$$
\hat{K}_{\mathrm{pw}}^{s}=\left(\frac{r-\varphi}{r}\right)^{\beta} \bar{K}_{\mathrm{pw}}^{s}=\left(\frac{S_{r \mathrm{O}}-S_{r \mathrm{C}}}{S_{r \mathrm{O}}-S_{r \mathrm{C}^{\prime}}}\right)^{\beta} \bar{K}_{\mathrm{pw}}^{s}=\left(\frac{S_{r \mathrm{~d}}-S_{r \mathrm{C}}}{S_{r \mathrm{~d}}-S_{r \mathrm{w}}}\right)^{\beta} \bar{K}_{\mathrm{pw}}^{s}
$$

In the $e: S_{r}$ projection plane, hysteresis still exists during cyclical drying-wetting processes. The same procedure can be adopted to deduce $\bar{K}_{\mathrm{pd}}^{e}, \bar{K}_{\mathrm{pw}}^{e}, \hat{K}_{\mathrm{pd}}^{e}$ and $\hat{K}_{\mathrm{pw}}^{e}$.

In summary, equations for main drying/wetting scanning curves in two projection planes are proposed. For any drying/wetting scanning curve in three-dimensional space, its increment of degree of saturation is attributed to both the 
increment of suction and that of void ratio. Therefore, incremental equations for main drying/wetting scanning curves in the $S_{\mathrm{r}}: s: e$ space can be written as:

for a drying process:

$$
\mathrm{d} S_{r}=\hat{K}_{\mathrm{pd}}^{e} \mathrm{~d} e+\hat{K}_{\mathrm{pd}}^{s} \mathrm{~d} s
$$

for a wetting process:

$$
\mathrm{d} S_{r}=\hat{K}_{\mathrm{pw}}^{e} \mathrm{~d} e+\hat{K}_{\mathrm{pw}}^{s} \mathrm{~d} s
$$

After determining equations for main drying/wetting scanning curves, the scanning curve corresponding to a single point can be obtained from these equations if any current position in the $S_{\mathrm{r}}: s: e$ space is provided.

\section{Model verification and analysis}

Experimental data are adopted to validate the applicability of the model deduced earlier, and the verification is grouped into calibrating model parameters and testing the predictive function of the model. Experimental data include statistics of main drying/wetting curves, bounding curves and scanning curves during drying-wetting cycles under simple or complex paths and so forth.

\subsection{Verification of effects of deformation for bentonite/kaolin mix}

During isotropic loading tests, the decrease of void ratio will contribute to the increase of degree of saturation when suction is constant. This section adopts the relationship between degree of saturation and void ratio at constant suction and under isotropic loading condition to validate the model's performance on prediction in the $S_{\mathrm{r}}: e$ plane[27]. Model parameters $a_{\mathrm{w}}, b_{\mathrm{w}}, n_{\mathrm{w}}$ are $1.132,0.039$ and 0.417 , respectively. Fig.4 presents the comparison between experimental results of bentonite/kaolin mix and the model's predicted results.

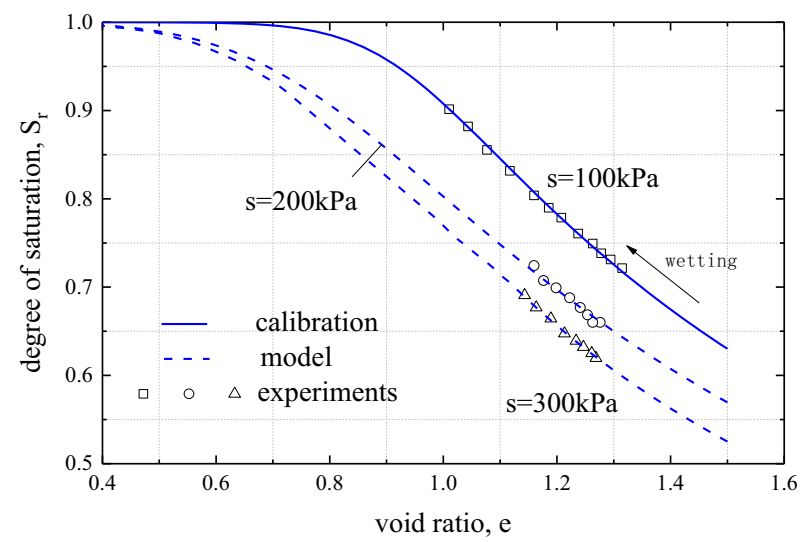

Fig.4. Comparison between experimental data obtained from the test of the relationship between $S_{\mathrm{r}}$ and $e$ and model prediction of main wetting curves at constant suction
At constant suction, the decrease of void ratio induced by loading lead to the increase of degree of saturation. At constant void ratio, degree of saturation climbs while suction goes down (see Fig.4). Obviously, at constant suction, results of degree of saturation obtained from the model are satisfactorily consistent with experimental results (in the $S_{\mathrm{r}}$ :e plane).

\subsection{Verification of effects of both hysteresis and deformation for pearl clay}

In this section, tests conducted by Sun are adopted[28]. These tests performed main drying/wetting paths and cyclical drying-wetting scanning paths on two samples of pearl clay whose void ratios are 1.08 and 1.78 , respectively. $a_{\mathrm{d}}, b_{\mathrm{d}}, n_{\mathrm{d}}, a_{\mathrm{w}}, b_{\mathrm{w}}, n_{\mathrm{w}}$ and $\beta$ are $28690,1.563,0.9228,3.018$, $0.3617,1.659$ and 5.062 , respectively. The change of void ratio is recorded during drying-wetting cycles, based on which the model's performance is validated.

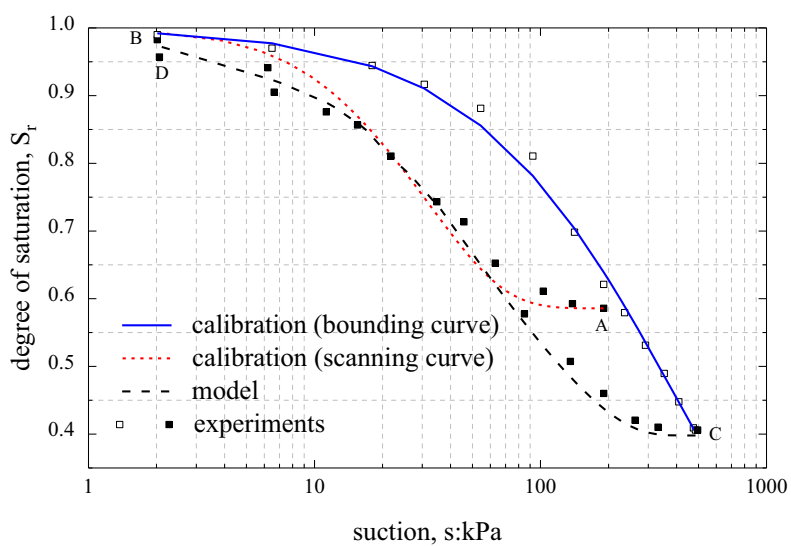

(a) $e_{0}=1.08$

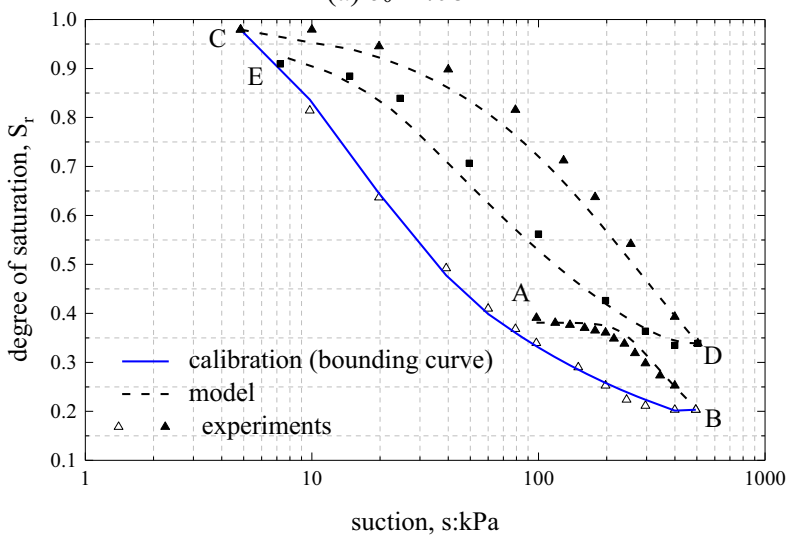

(b) $e_{0}=1.78$

Fig. 5. Comparison between model prediction and results from drying-wetting cycles performed on Pearl clay compacted at different void ratios. 


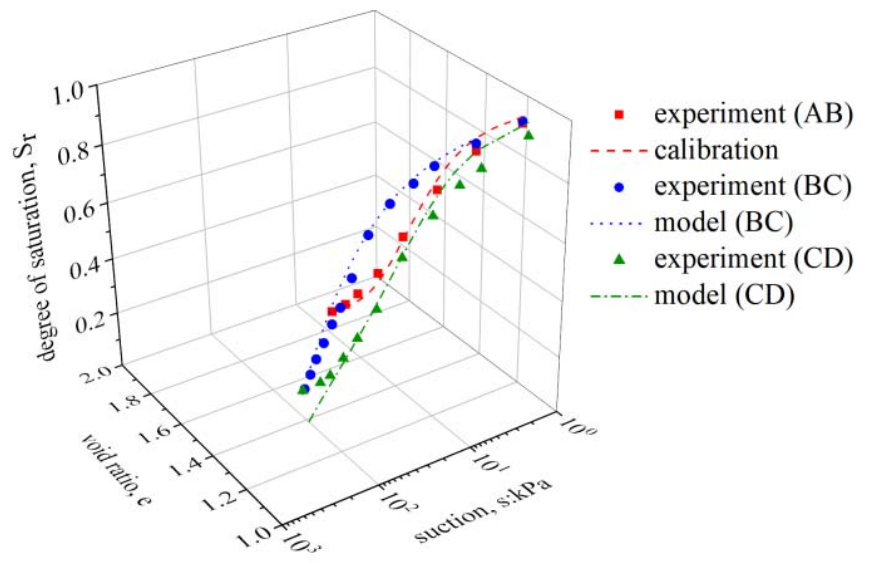

Fig.6. Comparison between model and experiments for samples with $e_{0}=1.08$ in three-dimensional space.

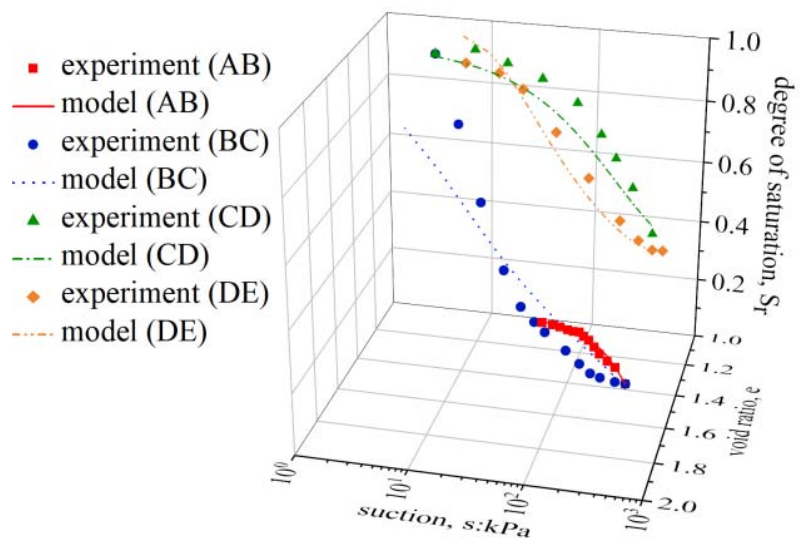

Fig.7. Comparison between model and experiments for samples with $e_{0}=1.78$ in three-dimensional space.

For the sample with $e_{0}=1.08$, its initial value of suction is $192 \mathrm{kPa}$. The test starts with a wetting path to $s=2 \mathrm{kPa}(\mathrm{AB})$, then the sample is dried to $s=490 \mathrm{kPa}(\mathrm{BC})$, after which a similar wetting process is conducted until $s=2 \mathrm{kPa}(\mathrm{CD})$. The whole test includes two wetting paths and one drying path (see Fig.5(a)).

For the sample with $e_{0}=1.78$, its initial value of suction is $98 \mathrm{kPa}$. The process begins with the drying of the sample to $s=490 \mathrm{kPa}(\mathrm{AB})$, then a wetting path is implemented until $s=5 \mathrm{kPa}(\mathrm{BC})$. In the following step, the sample is dried again to $s=490 \mathrm{kPa}(\mathrm{CD})$, and eventually, it is wetted to $s=7 \mathrm{kPa}$ (DE). The whole process contains two drying paths and two wetting paths (see Fig.5(b)).

Model parameters of main drying surfaces can be obtained through drying data from the section $\mathrm{BC}$ of the sample with $e_{0}=1.08$, and those of main drying surfaces can be attained by wetting data from the section $\mathrm{BC}$ of the sample with $e_{0}=1.78$.

Figure 5 presents both experimental and fitting results at two different void ratios in the $S_{\mathrm{r}}: s$ projection plane. Experimental results can be reproduced well by the model within the range of high suction. In particular, the model successfully represents significant and accurate hysteresis. During drying, the increase of suction or volumetric expansion of soil contributes to the irrevocable decrease of degree of saturation, which means water is discharged from pores while air enters. During wetting, the decrease of suction or volumetric shrinkage leads to the irreversible increase of degree of saturation, which means the soil absorbs water and air is expelled.

Verification of the three-dimensional soil-water characteristic surfaces is conducted by comparing with experimental results. For the sample of pearl clay with $e_{0}=1.08$, Fig. 6 presents its experimental results of bounding surfaces and scanning curves during drying-wetting cycles. Experimental points in scanning curves are all located between main drying curves and main wetting curves. The change of $S_{\mathrm{r}}$ in bounding curves is correlated to both deformation and suction, and the decrease of void ratio and suction collaboratively induce the increase of $S_{\mathrm{r}}$ in scanning curves. Fig.6 also presents the comparison between the model prediction and experimental results, and it can be observed that the model reproduces experimental behaviours very well.

For the sample of pearl clay with $e_{0}=1.78$, Fig. 7 presents its experimental results of bounding surfaces and scanning curves during drying-wetting cycles. Experimental points in scanning curves are also located between main drying curves and main wetting curves. During drying-wetting cycles, it can be seen that void ration and suction in scanning curves are changing simultaneously. The model also performed well compared with experimental results (see Fig.7).

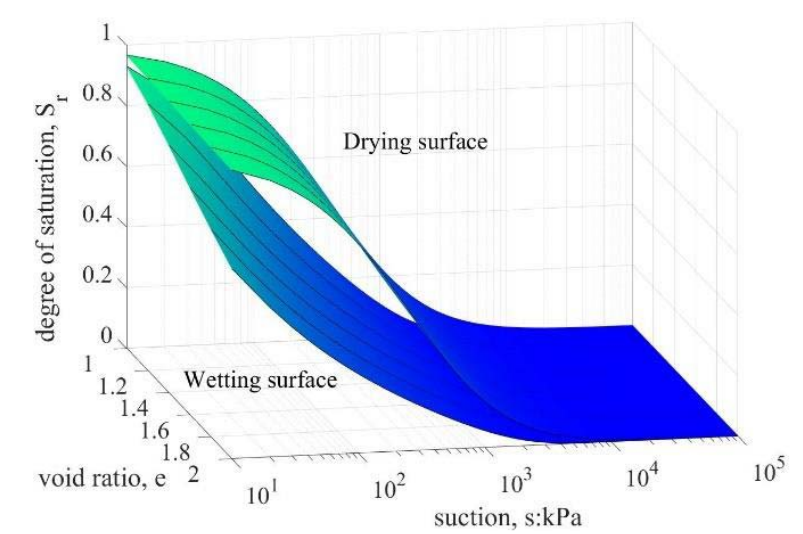

Fig.8. Soil-water characteristic bounding surfaces in the threedimensional space.

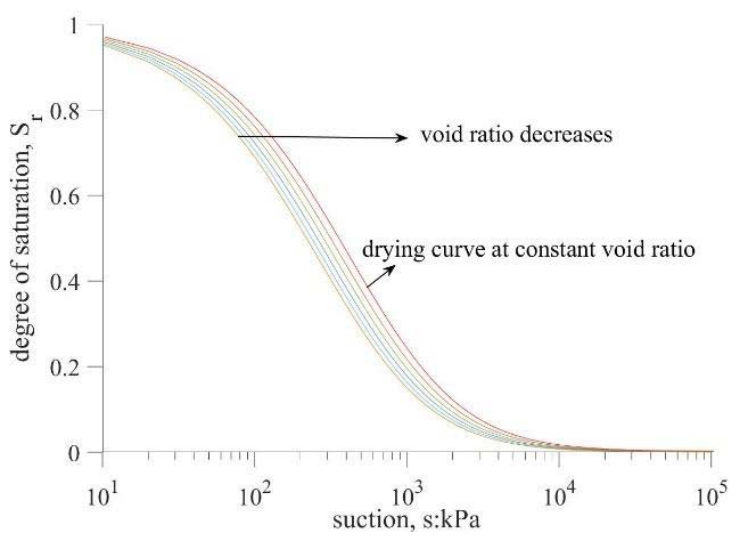

Fig.9. Soil-water characteristic curves at different constant void ratios in the $\left(s, \mathrm{~S}_{\mathrm{r}}\right)$ projection plane. 
Figure 8 presents the main wetting surface and the main drying surface in the three-dimensional space. It can be noticed that the larger the initial void ratio is, the bigger the size of hysteresis is, and vice versa. This illustrates that the water capacity of soil gets worse and hysteresis becomes more significant while the initial void ratio increases.

Soil-water characteristic curves with different constant void ratios are shown in Fig.9. With the decrease of void ratio, the curve moves right and the air entry value increases subsequently, which manifests consistency between the model prediction and present experimental results.

\section{Conclusions}

A three-dimensional soil-water characteristic surface model considering effects of deformation and hysteresis is developed in this paper, based on the plasticity bounding surface theory. Corresponding bounding surfaces and scanning curves are given in the $S_{\mathrm{r}}: e: s$ space, including their projective equations in different projection planes. The model has established a coupled relationship among $S_{\mathrm{r}}, e, s$ and has been adopted to reproduce hydraulic behaviours of soils.

The validation is conducted by utilizing data from bentonite/kaolin mix in the $S_{\mathrm{r}}: e$ plane and adopting experimental results of pearl clay in the $S_{\mathrm{r}}: s: e$ space. Experiments selected incorporate different kinds of soil and distinctive paths, and behaviours of soil samples can be satisfactorily reproduced by the model regardless of soil types and paths.

\section{Acknowledgements}

This research was financially supported by the National Natural Science Foundation of China (51722802, U1834206), Beijing Municipal Natural Science Foundation (8202038), Beijing Nova Program (Z181100006218005), and the Research Funds of Henan Provincial Department of Transportation (2017B4).

\section{References}

1. G. Q. Cai, A. N. Zhou, and D. C. Sheng, CAN GEOTECH J. 51(12), 1456-1467 (2014)

2. G. Q. Cai, C. G. Zhao, Y. Liu, and J. Li, SCI CHINA TECHNOL SC. 54(5), 1300-1314 (2011)

3. G. Q. Cai, C. G. Zhao, J. Li, and Y. Liu, J ZHEJIANG UNIV-SC A. 15(5), 364-373 (2014)

4. G. Q. Cai, P. Shi, X. Kong, C. Zhao, and W. J. Likos, ACTA GEOTECH, 1-9 (2019)

5. R. H. Brooks and A. T. Corey, T ASABE. 7(1), 26-0028 (1964)

6. M. T. Van Genuchten, SOIL SCI SOC AM J. 44(5), 892-898 (1980)

7. D. G. Fredlund and A. Xing, CAN GEOTECH J. 31(4), 521-532 (1994)

8. E. Romero, A. Gens, and A. Lloret, ENG GEOL. 54(12), 117-127 (1999)

9. D. Sun, Y. Gao, A. Zhou, and D. Sheng, B ENG GEOL
ENVIRON. 75(2), 781-791 (2016)

10. H. Q. Pham, D. G. Fredlund, and S. L. Barbour, CAN GEOTECH J. 42(6), 1548-1568 (2005)

11. S. Wheeler, R. Sharma, and M. Buisson, GEOTECHNIQUE. 53(1), 41-54 (2003)

12. E. Alonso, E. Romero, C. Hoffmann, and E. GarcíaEscudero, ENG GEOL. 81(3), 213-226 (2005)

13. R. Galindo-Aires, A. Lara-Galera, and S. Melentijevic, INT J GEOMECH. 19(6), 04019051 (2019)

14. Y. Tang, H. A. Taiebat, and A. R. Russell, INT J GEOMECH. 17(6), 04016142 (2016)

15. J. Liu, P. Chen, and W. Li, INT J GEOMECH. 18(7), 04018080 (2018)

16. Q. Zhai and H. Rahardjo, ENG GEOL. 163 144-152 (2013)

17. D. Gallipoli, S. J. Wheeler, and M. Karstunen, GEOTECHNIQUE. 53(1), 105-112 (2003)

18. M. Nuth and L. Laloui, COMPUT GEOTECH. 35(6), 835-844 (2008)

19. A. Tarantino, GEOTECHNIQUE. 59(9), 751-762 (2009)

20. S. Salager, M. S. El Youssoufi, and C. Saix, CAN GEOTECH J. 47(6), 609-622 (2010)

21. R. Hu, Y. Chen, and C. Zhou, CHIN J GEOTECH ENG. 35(8), 1451-1462 (2013)

22. A. Tsiampousi, L. Zdravkovic, and D. M. Potts, GEOTECHNIQUE. 63(2), 155-164 (2013)

23. Y. F. Dafalias, J ENG MECH. 112(9), 966-987 (1986)

24. X. S. Li, COMPUT GEOTECH. 32(2), 133-137 (2005)

25. C. F. Wei and M. M. Dewoolkar, WATER RESOUR RES. 42(7), (2006)

26. D. Manzanal, M. Pastor, and J. A. F. Merodo, INT J NUMER ANAL MET. 35(18), 1899-1917 (2011)

27. R. S. Sharma. PhD Dissertation, University of Oxford, UK (1998)

28. D. Sun, D. Sheng, H. Cui, and S. Sloan, INT J NUMER ANAL MET. 31(11), 1257-1279 (2007) 